\begin{tabular}{|c|c|c|c|c|c|}
\hline \multirow[b]{2}{*}{ Circuit } & \multirow[b]{2}{*}{ Memory Requirements } & \multicolumn{2}{|c|}{ Previous Algorithm [6] } & \multicolumn{2}{|c|}{ SPACK Algorithm } \\
\hline & & \begin{tabular}{|c|} 
Number of \\
Arrays Req'd \\
\end{tabular} & Utilization & \begin{tabular}{|c|} 
Number of \\
Arrays Req'd \\
\end{tabular} & Utilization \\
\hline $\begin{array}{l}\text { Variable Length } \\
\text { CODEC }\end{array}$ & $\begin{array}{l}\text { one } 768 \times 16, \text { two } 32 \times 7, \\
\text { one } 512 \times 1\end{array}$ & $\overline{77}$ & $\overline{446.2 \%}$ & $\overline{5}$ & $64.7 \%$ \\
\hline $\begin{array}{l}\text { Discrete Cosine } \\
\text { Transform Chip }\end{array}$ & two $16 \times 16$ & 4 & $3.13 \%$ & 2 & $6.25 \%$ \\
\hline $\begin{array}{l}\text { Video } \\
\text { Compression }\end{array}$ & $\begin{array}{l}\text { one } 24 \times 112 \text { (dual port) } \\
\text { one } 16 \times 96 \text { (dual port) }\end{array}$ & 26 & $3.97 \%$ & 20 & $5.16 \%$ \\
\hline $\begin{array}{l}\text { Encryption } \\
\text { Circuit }\end{array}$ & one $256 \times 16$ & 2 & $50 \%$ & 1 & $100 \%$ \\
\hline $\begin{array}{l}\text { Robot } \\
\text { Controller }\end{array}$ & one $172 \times 20$ & 3 & $28 \%$ & 2 & $42 \%$ \\
\hline Filter & $\begin{array}{l}\text { two } 8 \times 24 \text { (dual port) } \\
\text { one } 320 \times 24\end{array}$ & 9 & $21.9 \%$ & 9 & $21.9 \%$ \\
\hline $\begin{array}{l}\text { Neural Network } \\
\text { Chip 1 }\end{array}$ & one $160 \times 8$, one $32 \times 8$ & 2 & $18.8 \%$ & 1 & $37.5 \%$ \\
\hline $\begin{array}{l}\text { Neural Network } \\
\text { Chip } 2\end{array}$ & $\begin{array}{l}\text { one } 1310 \times 24, \\
\text { one } 1024 \times 16\end{array}$ & 13 & $89.8 \%$ & 13 & $89.8 \%$ \\
\hline $\begin{array}{l}\text { DMA Chip } \\
\text { for LAN }\end{array}$ & $\begin{array}{l}\text { one } 15 \times 24, \text { one } 16 \times 4, \\
\text { one } 256 \times 32\end{array}$ & 8 & $26.3 \%$ & 4 & $52.6 \%$ \\
\hline $\begin{array}{l}\text { Translation Look- } \\
\text { aside Buffer }\end{array}$ & $\begin{array}{l}\text { two } 256 \times 59, \\
\text { one } 16 \times 18 \text { (dual port) }\end{array}$ & 19 & $39.2 \%$ & 11 & $67.7 \%$ \\
\hline $\begin{array}{l}\text { Proof-of-Concept } \\
\text { Viterbi Decoder }\end{array}$ & $\begin{array}{l}\text { three } 128 \times 8, \\
\text { one } 28 \times 3 \text { (dual port) }\end{array}$ & 4 & $19.3 \%$ & 3 & $25.7 \%$ \\
\hline $\begin{array}{l}\text { Image } \\
\text { Backprojector }\end{array}$ & two $128 \times 22$ & 6 & $22.9 \%$ & 3 & $45.8 \%$ \\
\hline $\begin{array}{l}\text { DSP Control } \\
\text { Unit }\end{array}$ & $\begin{array}{l}\text { one } 1024 \times 32, \text { one } 128 \times 16, \\
\text { three } 64 \times 16, \text { two } 24 \times 16\end{array}$ & 20 & $47.2 \%$ & 14 & $67.4 \%$ \\
\hline $\begin{array}{l}\text { Vector } \\
\text { Processing } \\
\text { Unit }\end{array}$ & $\begin{array}{l}\text { two } 256 \times 9, \text { two } 256 \times 8, \\
\text { three } 128 \times 9, \\
\text { three } 128 \times 16 \text { (dual port) }\end{array}$ & 18 & $24.8 \%$ & 12 & $37.2 \%$ \\
\hline $\begin{array}{l}\text { Communications } \\
\text { Circuit } 1\end{array}$ & six $88 \times 8$, one $64 \times 24$ & 9 & $15.6 \%$ & 5 & $28.1 \%$ \\
\hline $\begin{array}{l}\text { Communications } \\
\text { Circuit } 2\end{array}$ & three $736 \times 16$ & 12 & $71.9 \%$ & 9 & $95.8 \%$ \\
\hline $\begin{array}{l}\text { Communications } \\
\text { Circuit } 3\end{array}$ & $\begin{array}{l}\text { four } 368 \times 16 \\
\text { one } 736 \times 16\end{array}$ & 12 & $71.9 \%$ & 11 & $78.4 \%$ \\
\hline $\begin{array}{l}\text { Communications } \\
\text { Circuit } 4\end{array}$ & $\begin{array}{l}\text { two } 1620 \times 3, \text { two } 168 \times 12, \\
\text { two } 366 \times 11\end{array}$ & 12 & $44.4 \%$ & 9 & $59.1 \%$ \\
\hline $\begin{array}{l}\text { Communications } \\
\text { Circuit } 5\end{array}$ & one $192 \times 12$ & 2 & $28.1 \%$ & 1 & $56.3 \%$ \\
\hline Average & & 9.89 & $35.4 \%$ & 7.10 & $51.7 \%$ \\
\hline
\end{tabular}

Table 2. Experimental Results 
8. J. Cong and S. Xu, "Technology mapping for FPGAs with embedded memory blocks," in Proceedings of the ACM/SIGDA International Symposium on FieldProgrammable Gate Arrays, pp. 179-187, February 1998.

9. Altera Corporation, "Implementing RAM functions in FLEX $10 \mathrm{~K}$ devices." Technical Note, Nov. 1995.

10. P. K. Jha and N. D. Dutt, "Library mapping for memories," in Proceedings of the 1997 European Design and Test Conference, March 1997.

11. D. Karchmer and J. Rose, "Definition and solution of the memory packing problem for field-programmable systems," in Proceedings of the IEEE International Conference on Computer-Aided Design, pp. 20-26, 1994.

12. H. Schmit and D. Thomas Jr., "Address generation for memories containing multiple arrays," IEEE Transactions on Computer-Aided Design of Integrated Circuits and Systems, vol. 17, May 1998.

13. P. R. Panda and N. D. Dutt, "Behavioral array mapping into multiport memories targeting low power," in Proceedings of the 10th International Conference on VLSI Design, Jan 1997.

14. M. Balakrishnan, A. Majumdar, D. Banerji, J. Linders, and J. Majithia, "Allocation of multiport memories in datapath synthesis," IEEE Transactions on Computer-Aided Design, vol. 7, April 1988. 
wasted (because each physical array could not be completely filled with logical memories). Using the previous algorithm, the utilization is only $35.4 \%$ averaged

over all circuits. SPACK results in a significantly higher utilization of $51.7 \%$.

\section{Conclusions}

In this paper, we have presented a new logical-to-physical mapping algorithm that targets FPGAs with dual-port embedded arrays. The purpose of the algorithm is to map the memories required by a circuit to the physical FPGA memory resources. This is an important problem, since an implementation of a user's memory that requires even one more physical array than necessary could very easily cause a circuit to not fit on a given FPGA.

Previous work has studied FPGAs with single-port embedded arrays. Most current FPGAs, however, contain dual-port arrays. We have shown that by explicitly taking advantage of the dual-port nature of these arrays, our algorithm produces considerably more efficient implementations of the memory parts of circuits. Specifically, we have shown that under the right conditions, we can pack two single-port user memories (or parts of two single-port user memories) into a dual-port array. Our algorithm results in memory implementations that use, on average, $28 \%$ fewer arrays than an algorithm that does not take advantage of the dual-port arrays in this way.

\section{Acknowledgments}

This work was supported by Cypress Semiconductor, the Natural Sciences a nd Engineering Research Council of Canada, and UBC's Centre for Integrated Computer Systems Research.

\section{References}

1. Altera Corporation, Datasheet: FLEX 1OK Embedded Programmable Logic Family, May 1998.

2. Altera Corporation, Datasheet: FLEX 1OKE Embedded Programmable Logic Family, August 1998.

3. Xilinx, Inc., "Virtex: Our new million-gate 100-MHz FPGA technology." XCell: The Quarterly Journal for Xilinx Programmable Logic Users, First Quarter 1998.

4. Actel Corporation, Datasheet: Integrator Series FPGAs: $40 M X$ and $42 M X$ Families, April 1998.

5. Lattice Semiconductor Corporation, Datasheet: ispLSI and pLSI 6192 High Density Programmable Logic with Dedicated Memory and Register/Counter Modules, July 1996.

6. S. J. E. Wilton, Architectures and Algorithms for Field-Programmable Gate Arrays with Embedded Memory. PhD thesis, University of Toronto, 1997.

7. S. J. E. Wilton, "SMAP: heterogeneous technology mapping for FPGAs with embedded memory arrays," in ACM/SIGDA International Symposium on FieldProgrammable Gate Arrays, pp. 171-178, February 1998. 


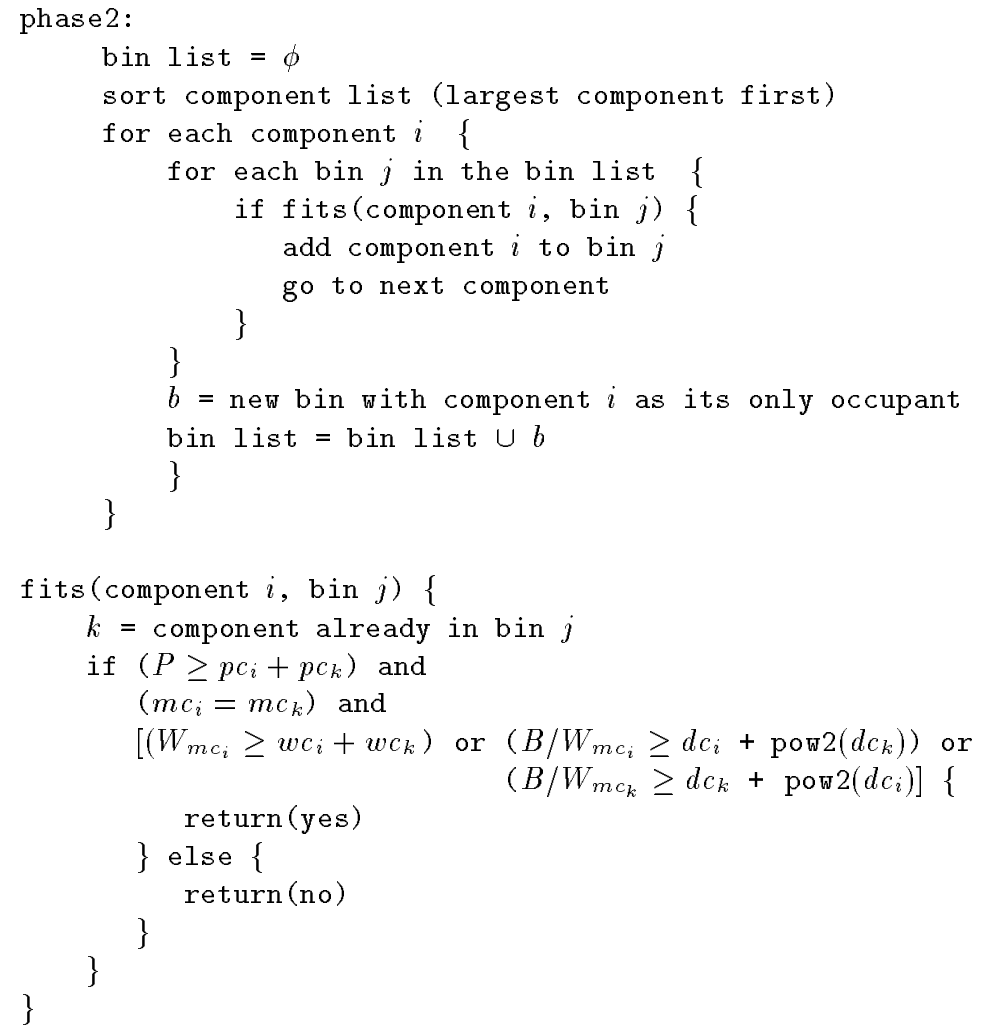

Fig. 5. Summary of Phase 2 of the Algorithm

Note that the purpose of Table 2 is not to compare FPGAs with single-port arrays to those with dual-port arrays. If the FPGA had only single-port arrays, many of the circuits in the table could not have even been implemented (unless the dual-port user memories were implemented by time-multiplexing two ports onto a single physical port). Rather, the purpose of the table is to show that when targeting FPGAs with dual-port arrays, our algorithm performs considerably better than the previous algorithm. Since we expect most future FPGAs to contain dual-port arrays, this is an important result.

The fourth and sixth columns of Table 2 show the utilization of the arrays. The utilization is defined as:

$$
\text { utilization }=100 \frac{\text { number of bits in logical memory configuration }}{\text { (number of bits in each array)(number of arrays used) }}
$$

A utilization of $100 \%$ means that every bit in the physical arrays was used, while a utilization lower than $100 \%$ means that some bits in the arrays were 
general, the following condition must be true to combine arrays:

$$
P \geq p c_{i}+p c_{j}
$$

Most FPGAs with dual-port arrays require that each port in a physical array be used in the same mode (eg. one port can not be used as a $4 \mathrm{Kx} 1$ while the other is used as a $512 \times 8$ ). Thus, one final constraint is that two components $i$ and $j$ can only be packed together if:

$$
m c_{i}=m c_{j}
$$

With these constraints, we can formulate the packing problem as a multidimensional bin-packing problem. The physical arrays are bins, and the components are the objects to be packed. In order for two components to be packed in the same bin, constraints 6 and 7 as well as either 4 or 5 must be satisfied. Figure 5 summarizes this phase of the algorithm.

\subsection{Phase 3: Wire together the Memories}

After phase 2, the components implementing each logical memory may be scattered among several physical arrays. The final step in the algorithm is to combine the arrays and connect them to the rest of the circuit. If the "horizontal" parti-

tioning was used in phase 1 , the address ports can be simply wired together. If the "vertical" partitioning was in phase 1, a multiplexor and decoder are needed to connect the components. Both of these techniques are described in [6] and [9] and so will not be discussed further here.

\section{Results and Discussion}

Our algorithm was implemented in a program called SPACK. To evaluate SPACK, we compare it to results obtained from the algorithm presented in [6]. That algorithm maps single-port logical memories to single-port arrays. Each logical memory is broken into components and each component is implemented by a single physical array (this is the same as our algorithm, without Phase 2). Although all logical memories considered in [6] were single-port, the algorithm will support dual-port memories, as long as the physical arrays are dual-port. Thus, we can compare it directly with our algorithm using benchmark circuits containing both single and dual-port logical memories.

Table 2 shows the results from SPACK and the previous algorithm for 19 benchmark circuits. The circuits and their logical memory configurations are shown in the first two columns of Table 2. Each circuit was mapped to 4Kbit physical arrays, each of which can be used as a $4 \mathrm{Kx} 1,2 \mathrm{Kx} 2,1 \mathrm{Kx} 4$, or $512 \mathrm{x} 8$. The number of arrays required to implement each benchmark using each algorithm is shown in the third and fifth columns of the table. Averaged over all benchmark circuits, the previous algorithm required 9.89 arrays, while SPACK required only 7.1 arrays. 


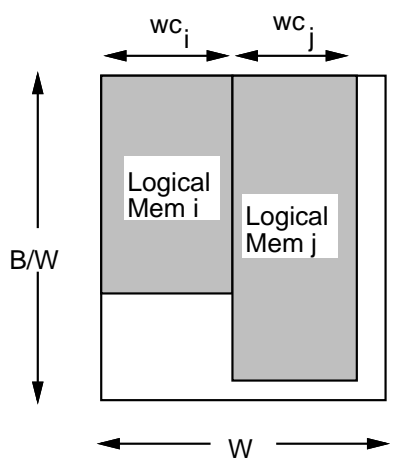

a)

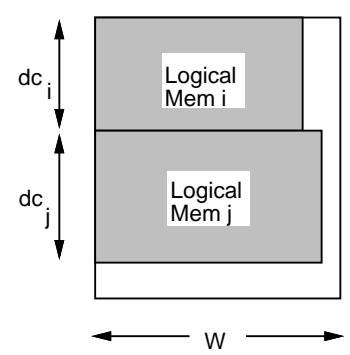

b)

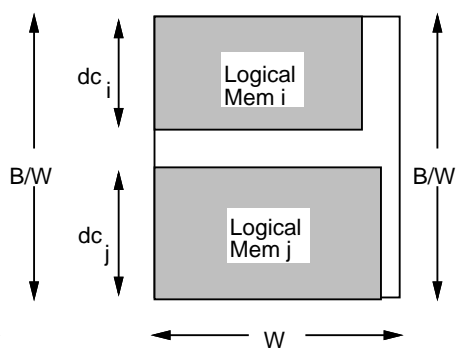

c)

Fig. 4. Combining Single-Port Logical Memories into Dual-Port Physical Arrays

Figure 4(b) shows how two components $i$ and $j$ can be combined "vertically". In this case, $d c_{i}$ of the words are used to implement component $i$ and $d c_{j}$ are used to implement component $j$. By accessing words 0 to $d c_{i}-1$ through one port and words $d c_{i}$ to $d c_{i}+d c_{j}-1$ through the other port, we can access each component independently. A straightforward implementation of this, however, would require an adder on the path feeding the second address port, since an offset of $d c_{i}$ must be added to the second component's address. This adder would be on the critical path of the memory access, which could slow down the circuit. We can eliminate the need for an adder as long as the following condition holds:

$$
B / W \geq d c_{i}+\operatorname{pow} 2\left(d c_{j}\right)
$$

where pow 2( $x)$ means $x$ is "rounded-up" to the next highest power-of-two.

As long as condition 5 holds, we can pack the components as shown in Figure $4(\mathrm{c})$. Component $i$ is implemented starting at word 0 , so no adder is needed on the first address port. Component $j$ is implemented in the top pow $2\left(d c_{j}\right)$ words. Then, $\log _{2}\left(\right.$ pow $2\left(d c_{j}\right)$ ) address lines in the second address port can be used to address component $j$, while the remaining lines in the second address port are set to ' 1 '. In this way, both memories can be accessed independently, and no adder is needed on either address port. The example in Figure 3(b) was implemented in this way.

Note that condition 5 is sufficient but not necessary. By employing the addressing techniques in [12], the constraint could be relaxed somewhat. Experimentation has shown, however, that for our problem, these more complex addressing schemes rarely lead to a final implementation that uses fewer memory arrays.

The above discussion has assumed that both components are single-ported. If either component requires two access ports, then it can not be packed with any other component, and must be implemented in its own physical array. In 


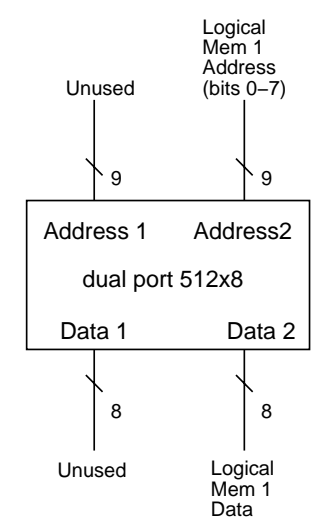

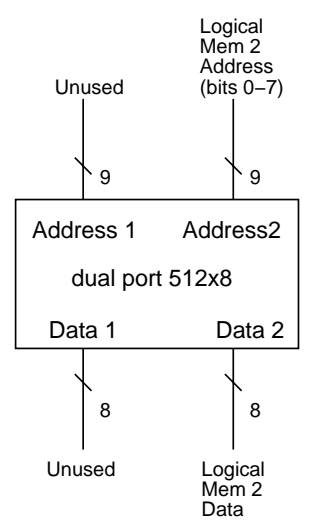

a)

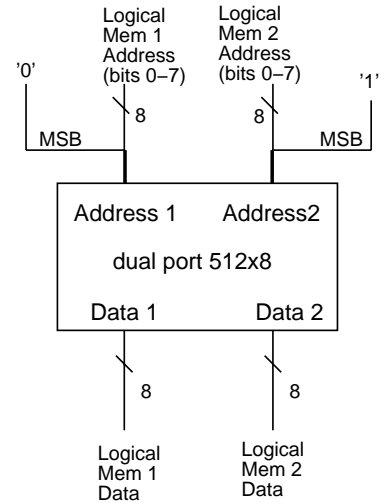

b)

Fig. 3. Two ways of implementing two single-port $192 \times 8$ logical memories

192x8 logical memories using an architecture with $B=4096$ and $P=2$. Phase 1 would create two components, each $192 \times 8$. If these components were implemented directly, two memory arrays would be required, as shown in Figure 3(a). Even though the original logical memory configuration only consists of 3072 bits, a total of 8192 bits (two physical arrays) are used to implement it. An alternative implementation is shown in Figure 3(b); in this implementation, each logical memory is mapped to a portion of a single array, and one of the array's two ports is used for each logical memory. The upper order address bit of port 1 is tied to 0 , while the upper order address bit of port 2 is tied to 1 . This ensures that each port sees a different $256 \times 8$ portion of the physical array. Since both ports are independent, both logical memories can be accessed independently. This example illustrates the goal in phase 2: the components from phase 1 are packed into the available memory arrays such that the total number of required arrays is as small as possible.

Informally, two single-port components can be packed into a dual-port physical array "vertically" or "horizontally". Consider packing two arrays $i$ and $j$ "horizontally" as shown in Figure 4(a). In this case, the physical array is of width $W$, and the two components are of width $w c_{i}$ and $w c_{j}$. Each word in the memory contains $w c_{i}$ bits for component $i$ and $w c_{j}$ bits for component $j$. By supplying an address to the first port's address bus, and accessing data through bits 0 to $w c_{i}-1$ of the first port's data bus, the first component can be accessed. The second component can be accessed in the same way using the second port's address bus and bits $w c_{i}$ to $w c_{i}+w c_{j}-1$ of the second port's data bus. Since the ports are independent, both components can be accessed independently.

In order to combine arrays in this way, it is sufficient that:

$$
W \geq w c_{i}+w c_{j}
$$

where $W$ is the physical array width in the mode chosen to implement the components. 


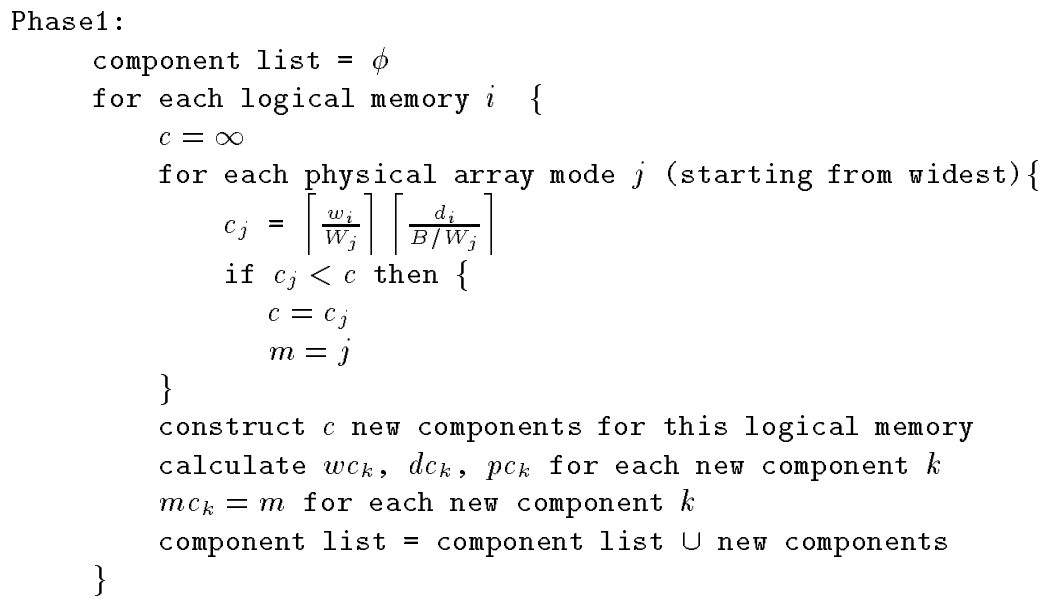

Fig. 2. Summary of Phase 1 of the Algorithm

In general, there are many ways to partition each logical memory. To simplify the task, we only consider partitions in which each component has the same $m c_{i}$ (that is, each component can be implemented by a physical array in the same mode). The partitions in Figures 1(a) and 1(b) would be considered, therefore, while the one in Figure 1(c) would not. Note that this only applies to components that make up a single logical memory. A logical memory configuration typically has several logical memories; components from different logical memories may correspond to different physical array modes.

Given this assumption, the number of components required to implement a logical memory $i$ using physical array mode $j$ is:

$$
c=\left\lceil\frac{w_{i}}{W_{j}}\right\rceil\left\lceil\frac{d_{i}}{B / W_{j}}\right\rceil
$$

To find the partition that results in the smallest $c$, we cycle through all possible array modes and choose the best result. This partitioning is done independently for each logical memory in the logical memory configuration. This is summarized in Figure 2.

\subsection{Phase 2: Bin Packing}

Given the list of components found in phase 1, it is possible to implement the logical memory configuration directly by using one physical array for each component. As will be shown in Section 4, this often results in very poor utilization of the memory arrays. As an example, consider implementing two single-port 


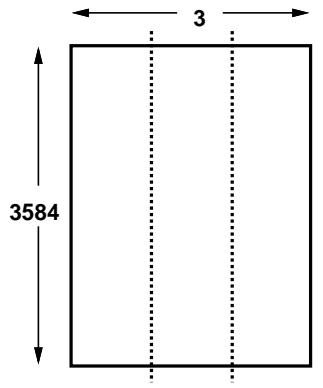

a)

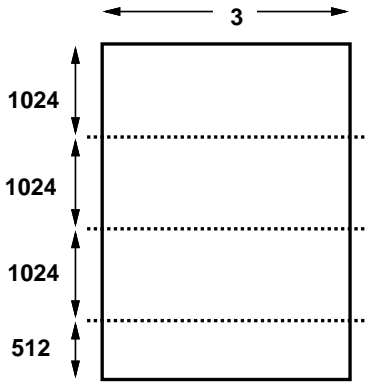

b)

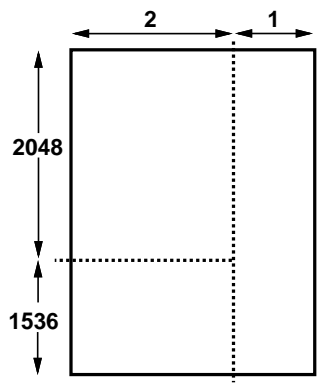

c)

Fig. 1. Three ways to partition a $3584 \times 3$ logical memory

\section{Logical-to-Physical Mapping Algorithm}

In this section, our new logical-to-physical mapping algorithm is described. The algorithm consists of three phases: during the first phase, the logical memories are broken into components, in the second phase, these components are packed into the physical arrays, and in the third phase, these physical arrays are wired together to implement the original logical memory configuration.

\subsection{Phase 1: Break Logical Memories into Components}

The first phase of the algorithm partitions each logical memory into several components, each of which is small enough to fit into a single physical array. Each component represents a portion of the bits in the original logical memory, and can be described by its width, $w c_{i}$, its depth, $d c_{i}$, and the number of ports required, $p c_{i}$. In order for the component to fit in a single physical array, $w c_{i}$ and $d c_{i}$ must satisfy the following inequalities:

$$
\begin{aligned}
w c_{i} & \leq W_{j} \\
d c_{i} & \leq B / W_{j}
\end{aligned}
$$

for some value of $j$ between 0 and $M-1$ (recall that a physical array can be used in one of $M$ modes, each of which has a different width/depth). The number of ports required by each component, $p c_{i}$, is the same as the number of ports required by the original logical memory. We also define a quantity $m c_{i}$ for each component which indicates the physical array mode(s) that can be used to implement this component.

As an example, Figure 1 shows three ways in which a $3584 \times 3$ logical memory could be broken into components. In each case, it is assumed that each physical array consists of 4096 bits $(B=4096)$ and can be used as a $4096 \times 1,2048 \times 2$, $1024 \times 4$, or a $512 \times 8$. In Figure 1(a), $w c_{i}=1$ for each component, while in Figure 1(b), all components have $w c_{i}=3$. In Figure 1, one of the components has $w c_{i}=1$, while the others have $w c_{i}=2$. 
used in many architectures today, we feel that true dual-port memories will be available in future devices. Thus, we focus our efforts on studying algorithms that target true dual-port memories.

\subsection{User Circuit Assumptions}

It is assumed that the user circuit to be implemented on the FPGA contains both logic and memory portions. In this paper, we are only concerned with the memory portion.

We assume that the memory portion of the circuit consists of $l$ independent user memories. We refer to each of these user memories as a logical memory. The set of all logical memories required for a circuit will be referred to as that circuit's logical memory configuration. The depth of logical memory $k(0 \leq k \leq$ $l-1$ ) will be denoted $d_{k}$, the width of logical memory $k$ will be denoted $w_{k}$, and the number of ports required by logical memory $k$ (maximum number of simultaneous accesses to memory $k$ ) will be denoted $p_{k}$. Unlike [6], we allow user memories that require either one or two ports. These parameters are summarized in the bottom half of Table 1.

\subsection{Problem Statement}

The problem studied in this paper can be stated as follows:

Given: 1. An FPGA architecture described by $B, M$ and $W_{i}(0 \leq$ $i<M$ ) as described in Subsection 2.1 (this paper only considers architectures with $P=2$ ),

2. A Logical Memory Configuration described by $l, d_{k}$, $w_{k}$, and $p_{k}(0 \leq k<l)$, as described in Subsection 2.2 (in this paper, $1 \leq p_{k} \leq 2$ for all $k$ ),

Find: An implementation of the logical memory configuration using $n$ embedded memories.

Such that: $n$ is as small as possible.

Note that the goal is to implement the logical memory configuration using as few physical arrays as possible. In an FPGA with $N$ arrays, it may appear that minimizing the number of arrays required to implement the logical memory configuration is immaterial, as long as the implementation requires $N$ or fewer arrays. Minimizing the number of arrays is important, however, since the remaining arrays can be configured as ROMs, and be very efficiently used to implement the logic part of the user circuit $[7,8]$. The fewer arrays that are used to implement memory, the more that will be available to implement logic. 


\begin{tabular}{|c|l|}
\hline Parameter & Meaning \\
\hline$N$ & Number of Arrays \\
$B$ & Bits per Array \\
$P$ & Ports per Array \\
$M$ & Number of Modes for each array \\
$W_{i}$ & Data width of array in mode $i$ \\
\hline$l$ & Number of Logical Memories \\
$d_{k}$ & Depth of Logical Memory $k$ \\
$w_{k}$ & Width of Logical Memory $k$ \\
$p_{k}$ & Ports in Logical Memory $k$ \\
\hline
\end{tabular}

Table 1. Architectural and Circuit Parameters

those obtained by simply extending a previous algorithm that was developed to target single-port arrays.

\section{Problem Definition}

In this section, we first describe our assumptions regarding the target FPGA architecture and the user circuit that is to be mapped, and then present a precise definition of the Logical-to-Physical Memory Mapping problem.

\subsection{Architectural Framework}

The top half of Table 1 summarizes the parameters that define the FPGA embedded memory array architecture. The number of embedded memory arrays is denoted by $N$, the number of bits in each array is denoted by $B$, the number of independent access ports in each array is denoted by $P$. Each array can be used in one of $M$ different modes; each mode has a different width and depth. The width of each array in mode $i$ is denoted $W_{i}$; the depth can be calculated as $B / W_{i}$. In the Altera FLEX10KE, $B=4096 \mathrm{bits}, P=2, M=4$, and $\left\{W_{0}, W_{1}, W_{2}, W_{3}\right\}=\{2,4,8,16\}$, meaning each array is dual-port and can be configured to be one of $2048 \times 2,1024 \times 4,512 \times 8$, or $256 \times 16$.

In this paper, we will only consider dual port arrays, ie. $P=2$. Note that some FPGA architectures, such as the Altera FLEX10KE, contain two independent ports, but one port is a dedicated read port and one port is a dedicated write port. This works well for many applications (such as a first-in first-out buffer that is used to temporarily hold data in a communication system), but there are many applications for which this is insufficient (a dual-port register file in a processor which must be read by two functional units simultaneously, for example). To implement these sorts of circuits, true dual-port memory arrays are required, in which the two accesses are independent, and either can be a read or write. With the increasing importance of embedded memory in FPGAs, and since true dualport arrays appear to be a natural evolution from the restricted dual-port model 
depth. As an example, the Altera 10KE devices contain between six and twenty 4 -Kbit blocks, each of which can be used as a $2 \mathrm{Kx} 2$, a $1 \mathrm{Kx} 4$, a $512 \mathrm{x} 8$, or a $256 \times 16$ array. ${ }^{1}$ These arrays can be combined to implement larger user memories.

The task of implementing the memories required by a user circuit using the FPGA embedded arrays is called logical-to-physical mapping [6]. Because of the large number of ways in which arrays can be combined, and because each array can be used in one of several modes (widths/depths), this problem is not trivial. Yet, it is vitally important - since each FPGA contains only a few memory arrays, a sub-optimal implementation that wastes even one memory array could very easily cause a circuit to not fit on a given FPGA. Even if the memory configuration does fit on the FPGA, minimizing the number of arrays needed to implement the storage part of the circuit is beneficial because unused memory arrays can be configured as ROM and used to implement logic $[7,8]$.

In $[6,9]$, logical-to-physical mapping for FPGAs with single-port embedded arrays (arrays in which only one access can be performed at a time) is discussed. Many recent FPGAs, however, contain dual-port arrays (so that two accesses can be performed by each array concurrently) [2-4]. Many applications require memories that can be accessed simultaneously by two separate subcircuits; these applications can most efficiently be implemented if the FPGA has dual-port arrays.

In this paper, a new logical-to-physical mapping algorithm that targets dualport arrays is presented. We show that this new algorithm results in much more efficient implementations than if we simply extend the techniques targeting single-port arrays [6,9]. The user circuits are assumed to consist of both single and dual-port user memories; our improvement is obtained by intelligently packing the single-port user memories into the dual-port physical arrays. Under the right conditions, each dual-port array can implement two single-port memories (or parts of two single-port memories).

Besides [6] and [9], little work as been done in this area. Jha and Dutt describe an algorithm to map logical memories to physical library elements [10], but do not consider the optimizations that are possible when the physical elements are dual-port. Karchmer and Rose show how user memories can be implemented by larger physical memory chips, but only consider single-port physical arrays [11]. Their work is also different in that they consider discrete memory devices, which do not have the variety of modes that FPGA memory arrays have. There has also been considerable work mapping variables to both single and dual port memories during high-level synthesis in an attempt to minimize the execution time of an algorithm [12-14]. None of these papers consider physical memories with the configurability of FPGA arrays, however.

This paper is organized as follows. Section 2 presents our assumptions regarding the FPGA architecture and the application circuits, and then gives a precise definition of the problem solved in this paper. Section 3 then describes our algorithm. Finally, Section 4 compares the results from our algorithm with

\footnotetext{
${ }^{1}$ In this paper, a $a \times b$ memory has $a$ words of $b$ bits each.
} 


\title{
Logical-to-Physical Memory Mapping for FPGAs with Dual-Port Embedded Arrays
}

\author{
William K.C. Ho and Steven J.E. Wilton \\ Department of Electrical and Computer Engineering \\ University of British Columbia, \\ Vancouver, B.C., Canada, \\ $\{$ williamh|stevew $\}$ Oece.ubc.ca \\ http://ww. ece.ubc.ca/ ${ }^{\sim}$ stevew
}

\begin{abstract}
On-chip storage has become critical in large FPGAs. This has led most FPGA vendors to include configurable embedded arrays in their devices. Because of the large number of ways in which the arrays can be combined, and because of the configurability of each array, there are often many ways to implement the memories required by a circuit. Implementing user memories using physical arrays is called logical-tophysical mapping, and has previously been studied for single-port FPGA memory arrays. Most current FPGAs, however, contain dual-port arrays. In this paper, we present a logical-to-physical algorithm that specifically targets dual-port FPGA arrays. We show that this algorithm results in $28 \%$ denser memory implementations than the only previously published algorithm.
\end{abstract}

\section{Introduction}

It has become clear that on-chip storage is critical in large FPGAs. As FPGAs grow, they are being used to implement entire systems, rather than the small logic subcircuits that have traditionally been targeted to FPGAs. One of the important differences between these large systems and smaller logic subcircuits is that the large systems often require storage. Although this storage could be implemented off-chip, on-chip storage has a number of advantages. Besides the obvious advantages of integration, on-chip storage will often lead to higher clock frequencies, since I/O pins need not be driven with each memory access. In addition, on-chip storage will relax I/O pin requirements, since pins need not be devoted to external memory connections. These advantages have led most FPGA vendors to produce architectures with significant amounts of on-chip storage.

Since the storage requirements of circuits vary widely, the FPGA memory architecture must be flexible enough to implement different numbers of independently addressable memories as well as different memory shapes and sizes. Many recent commercial devices, such as the Altera $10 \mathrm{~K}$ and $10 \mathrm{KE}$ devices $[1$, 2], the Xilinx Virtex FPGAs [3], the Actel 42MX [4], and the Lattice ispLSI 6192 FPGAs [5], provide several large arrays embedded into the FPGA. Each array can typically be used in one of several modes, each with a different width and 